\title{
Burden of nonalcoholic fatty liver disease in Canada, 2019-2030: a modelling study
}

\author{
Mark G. Swain MD, Alnoor Ramji MD, Keyur Patel MD PhD, Giada Sebastiani MD, \\ Abdel Aziz Shaheen MBBCh, Edward Tam MD, Paul Marotta MD, Magdy Elkhashab MD, \\ Harpreet S. Bajaj MD, Chris Estes MPH, Homie Razavi PhD
}

\section{Abstract}

Background: Nonalcoholic fatty liver disease (NAFLD) and nonalcoholic steatohepatitis (NASH) account for a growing proportion of liver disease cases, and there is a need to better understand future disease burden. We used a modelling framework to forecast the burden of disease of NAFLD and NASH for Canada.

Methods: We used a Markov model to forecast fibrosis progression from stage F0 (no fibrosis) to stage F4 (compensated cirrhosis) and subsequent progression to decompensated cirrhosis, hepatocellular carcinoma, liver transplantation and liver-related death among Canadians with NAFLD from 2019 to 2030. We used historical trends for obesity prevalence among adults to estimate longitudinal changes in the number of incident NAFLD cases.

Results: The model projected that the number of NAFLD cases would increase by $20 \%$ between 2019 and 2030 , from an estimated 7757000 cases to 9305000 cases. Increases in advanced fibrosis cases were relatively greater, as the number of model-estimated prevalent stage F3 cases would increase by $65 \%$, to 357000 , and that of prevalent stage $\mathrm{F} 4$ cases would increase by $95 \%$, to 195 000. Estimated incident cases of hepatocellular carcinoma and decompensated cirrhosis would increase by up to $95 \%$, and the number of annual NAFLD-related deaths would double, to 5600.

Interpretation: Increasing rates of obesity translate into increasing NAFLD-related cases of cirrhosis and hepatocellular carcinoma and related mortality. Prevention efforts should be aimed at reducing the incidence of NAFLD and slowing fibrosis progression among those already affected.

N onalcoholic fatty liver disease (NAFLD), defined by the presence of excessive liver fat in the absence of another causative factor, ${ }^{1}$ is recognized to cause cirrhosis and hepatocellular carcinoma..$^{2-4}$ Cases of NAFLD can be characterized as simple steatosis or nonalcoholic steatohepatitis (NASH), characterized by additional histologic features. Obesity rates are increasing in Canada, affecting over one-third of Canadians, ${ }^{5,6}$ and obesity is 1 component of metabolic syndrome, which is a risk factor for NAFLD. ${ }^{1,7}$ There is a growing disease burden associated with NAFLD, following the trajectory of increasing obesity in Canada and globally. ${ }^{8}$ Even if further increases in obesity in Canada are halted, NAFLD-related morbidity and mortality are projected to increase for decades.

Analyses of the disease and economic burden associated with NAFLD based on data in the existing literature have recently been reported. ${ }^{9-11}$ To our knowledge, there are currently no studies reporting estimates of NAFLD prevalence or disease burden in the general Canadian population, but estimates have been reported in selected populations ${ }^{12}$ in other areas. ${ }^{13,14}$ Given the substantial health and socioeconomic burden of NAFLD ${ }^{7}$ there is an increasing need to forecast the burden of disease. A modelling framework can provide a range of outcomes that can help in effective resource use and the development of strategies to prevent further increases in disease burden. The current analysis describes the results of such modelling to forecast the burden of disease of NAFLD and NASH for Canada, from 2019 to 2030.

Competing interests: Funding for this project was provided by Gilead Sciences. No other competing interests were declared.

This article has been peer reviewed.

Correspondence to: Chris Estes, cestes@cdafound.org

CMAJ Open 2020. DOI:10.9778/cmajo.20190212 


\section{Methods}

A Markov model (Appendix 1, Supplemental Figure S1, available at www.cmajopen.ca/content/8/2/E429/suppl/DC1) was built with the use of Microsoft Excel to estimate the number of NAFLD cases by disease stage in Canada beginning in $1950 .{ }^{15}$

\section{Model}

The model tracked the Canadian population by age group and gender over time, with adjustment for background mortality at every step. New NAFLD cases entered the model based on longitudinal adult obesity trends. We calculated progression of disease through fibrosis, ${ }^{16}$ decompensated cirrhosis, hepatocellular carcinoma and liver transplantation stages (Appendix 1, Supplemental Figure S1) after accounting for both all-cause mortality (with adjustment for excess cardiovascular and non-liver-cancer mortality) and liver mortality attributable to NAFLD. Fibrosis transition rates were varied by gender (males experience faster progression) and age (Appendix 1, Supplemental Tables S2 and S3).

We implemented a Delphi process in which expert consensus was used to develop key model inputs (Appendix 1, Supplemental Table S1). Experts were invited based on research expertise and represented multiple disease areas including hepatology and diabetology. In addition, the expert panel included representation from provinces representing a majority of the Canadian population.

\section{Population and mortality}

We organized the total Canadian population and background mortality rates by year, gender and 5 -year age groups. ${ }^{17}$ Increased background mortality among NAFLD cases was based on reported hazard ratios for all-cause mortality by fibrosis stage, ${ }^{18}$ adjusted to remove the impact of increased liverrelated mortality, ${ }^{19}$ as this was calculated as a separate progression in the model. ${ }^{2,20-22}$ After adjustment, there was no increased non-liver-related mortality in stages F0-F2; increased mortality ratios of 1.42 and 1.43 were applied to prevalent F3 and F4 cases, respectively (Appendix 1, Supplemental Table S2). For the oldest age groups (age $\geq 75 \mathrm{yr}$ ), no excess mortality was assumed given the already high mortality rates in this age group and reported decreases in the incremental impact of cardiovascular disease mortality among people aged 75 years or older. ${ }^{23}$

\section{New cases of nonalcoholic fatty liver disease}

We extrapolated the annual number of new NAFLD cases based on longitudinal changes in the prevalence of adults in different body mass index categories, with the assumption that relative increases in NAFLD prevalence would mirror changes in obesity prevalence ${ }^{24-26}$ (Appendix 1, Incidence [new cases] calculations). Owing to variations in cut-off levels for obesity that vary by race or ethnicity ${ }^{27}$ and differences in health risk by body mass index class, ${ }^{28}$ we calculated the prevalence of obesity as a weighted average using data for body mass index of 25 or greater for the population classified by 1996-2016 census data as South Asian, Chinese, Filipino, Southeast Asian, Korean or Japanese, ${ }^{29}$ and data for body mass index of 30 or greater for the remaining population. We estimated the total population with a body mass index of 25 or greater to be $7.3 \%$ of the total Canadian population in 2006, $14.2 \%$ in 2016 and, with linear trending, $19.0 \%$ in 2030.

Using the weighted average for the body mass index categories as described above, we extrapolated changes in obesity prevalence based on trending of data for the adult prevalence of body mass index of 25 or greater and of 30 or greater, ${ }^{24-26}$ where the adjusted obesity prevalence rate was estimated at 9.1\% in 1975 and $32.7 \%$ in 2014 (Appendix 1, Supplemental Figure S2).

\section{Prevalence of nonalcoholic fatty liver disease}

Among people aged 20 years or older in 2018, there was an assumed NAFLD prevalence rate of 25\% (Delphi range 22.5\%$27.5 \%$ ) based on expert consensus. The prevalence of NAFLD was assumed to decline among the youngest age groups ( $\leq 18 \mathrm{yr})$, who are often not included in studies based on the general population. ${ }^{30,31}$ We based the age and gender distribution of prevalent NAFLD cases on data from general population studies, in which prevalence increases with increasing age. ${ }^{30,32,33}$ Based on a cohort of patients with NAFLD in Calgary, we assumed that prevalence would be approximately equal between men and women. ${ }^{34}$ Owing to elevated mortality, we assumed that prevalence would naturally decline among the oldest age groups ( $\geq 80 \mathrm{yr}$ ), with peak prevalence occurring in late middle age.

\section{Liver transplantation}

Annual liver transplantation procedures were reported by Canadian Blood Services..$^{35}$ Based on expert input and review of diagnostic categories for transplant recipients, ${ }^{36}$ we estimated that about one-quarter of current liver transplantation procedures could be attributable to NAFLD or NASH.

\section{Statistical analysis}

We simulated disease progression by multiplying the total number of cases at a particular stage of the disease by a progression rate to the next stage, with adjustments for mortality at every step (Appendix 1, equation 1). We used Monte Carlo simulation to identify model inputs that accounted for the greatest variation in future disease burden and to produce 95\% uncertainty intervals for selected model outputs (Appendix 1 , Uncertainty and sensitivity analysis).

\section{Ethics approval}

Since this was a modelling study, no ethics approval was needed.

\section{Results}

\section{Nonalcoholic fatty liver disease}

Between 2019 and 2030, the number of model-estimated NAFLD cases in Canada would increase by 20\%, to 9305000 (uncertainty interval 8550 000-9 875000 ) (Figure 1). Accounting for population growth, the estimated prevalence would increase from $20.8 \%(19.1 \%-22.1 \%)$ to $22.9 \%(21.1 \%-$ $24.3 \%$ ), and the age-adjusted prevalence would increase from $20.8 \%(19.1 \%-22.1 \%)$ to $22.3 \%(20.5 \%-23.7 \%)$ (Table 1$)$. 
In 2019, the largest prevalent NAFLD age group would be 55-59 years, with 995000 estimated NAFLD cases. By 2030, the largest prevalent age group would be 65-69 years, with 1079000 predicted cases (Appendix 1, Supplemental Figure S3).
Over the same period, the unadjusted number of stage F0 NAFLD cases would increase by 15\%, to 7400000 (6 577000 $8048000)$. The number of prevalent stage $\mathrm{F} 1$ cases would increase by $30 \%$, to 807000 (542 000-1 123000$)$.
A

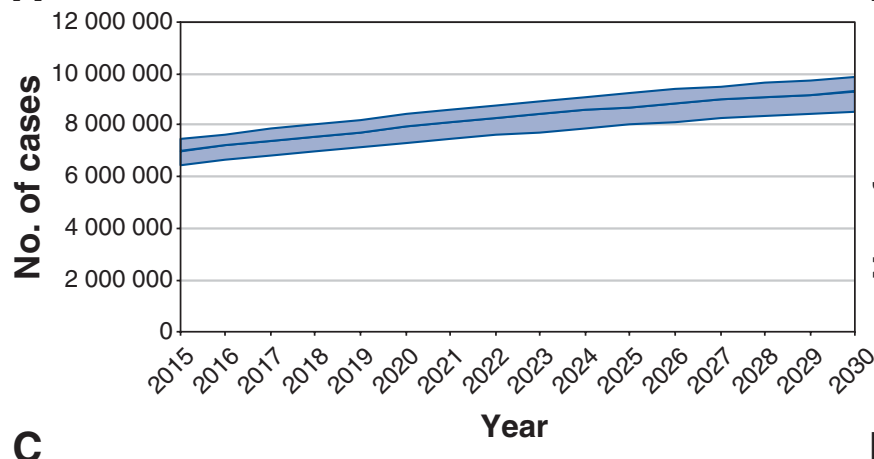

B

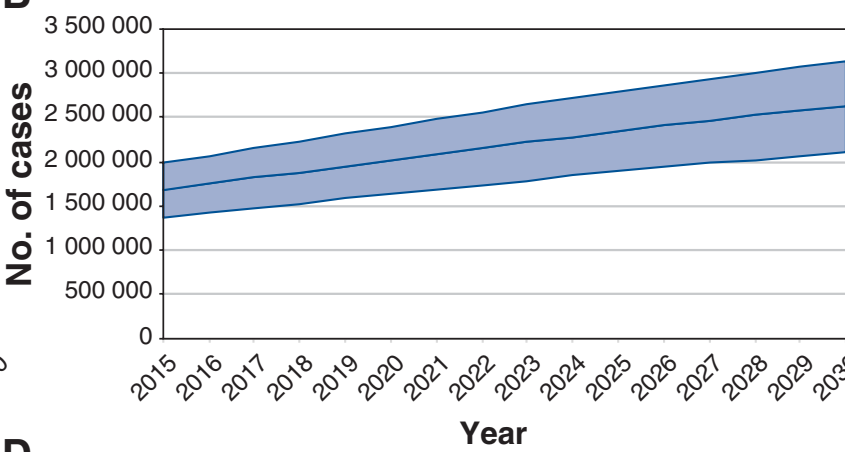

D

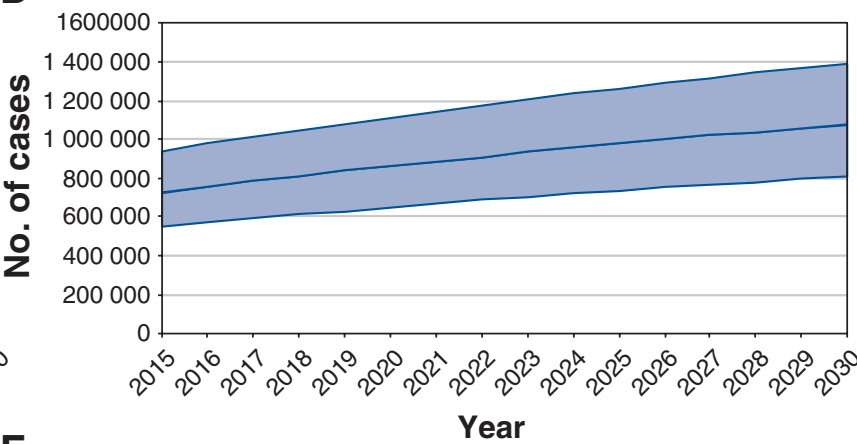

$\mathbf{F}$

E

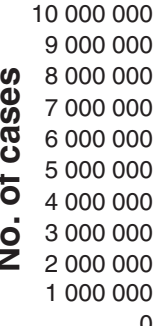

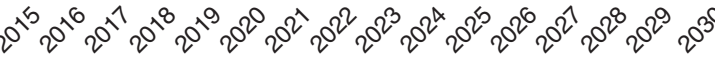

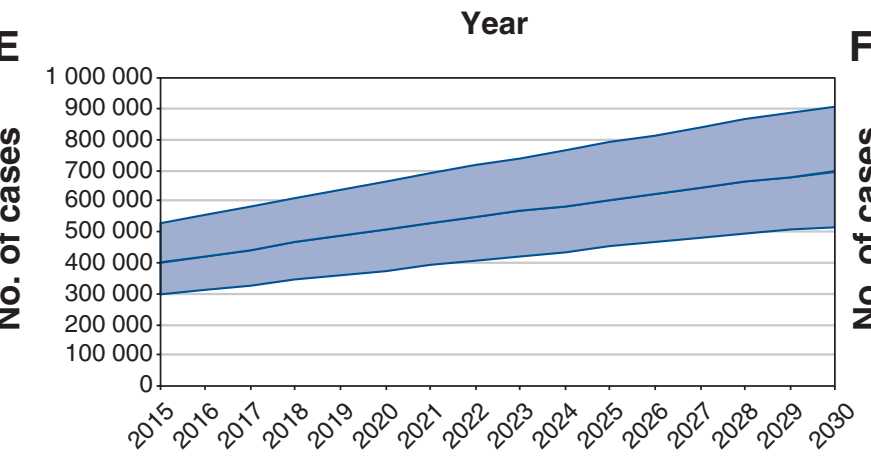

G

Year

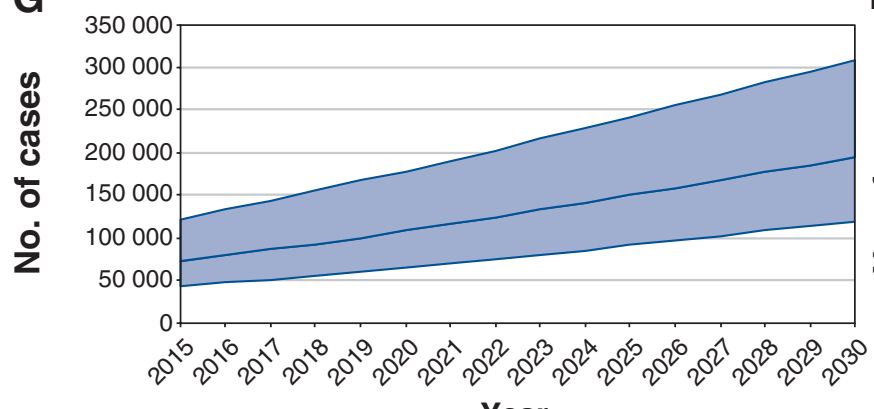

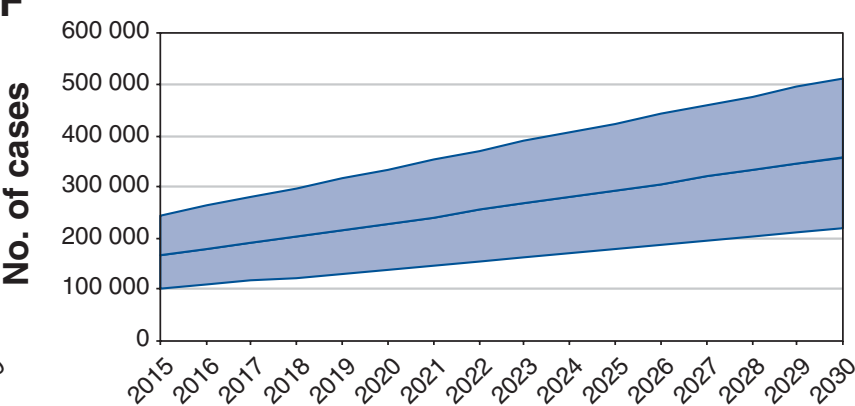

H

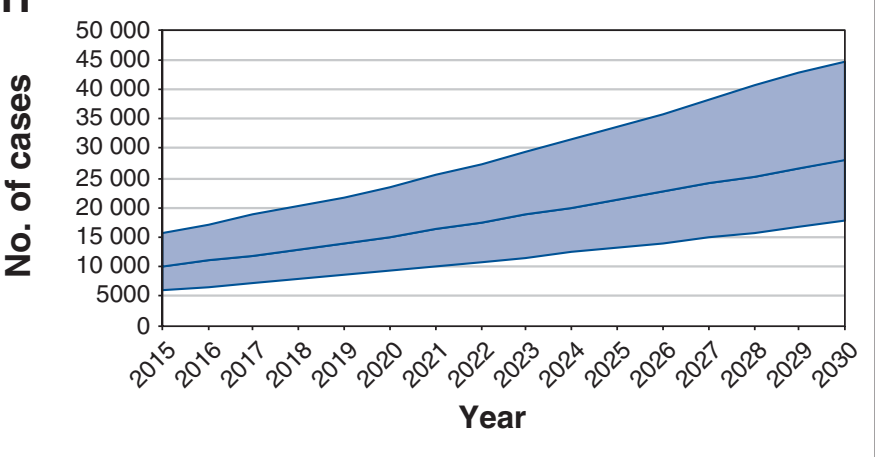

Figure 1: Model-estimated prevalent cases of nonalcoholic fatty liver disease (NAFLD) (A), nonalcoholic steatohepatitis (B), stage F0 NAFLD (C), stage F1 NAFLD (D), stage F2 NAFLD (E), stage F3 NAFLD (F), compensated cirrhosis NAFLD (G) and decompensated cirrhosis, hepatocellular carcinoma and liver transplantation related to NAFLD (H) for Canada, 2015-2030. Shaded areas represent 95\% uncertainty interval. 


\begin{tabular}{|c|c|c|c|c|}
\hline \multirow[b]{2}{*}{ Variable } & \multicolumn{4}{|c|}{ Year; no. (uncertainty interval)* } \\
\hline & 2019 & 2020 & 2025 & 2030 \\
\hline Population of Canada & 37280000 & 37603000 & 39173000 & 40618000 \\
\hline \multicolumn{5}{|c|}{ Model-estimated prevalent cases } \\
\hline NAFLD & $\begin{array}{c}7757000 \\
(7138000-8232000)\end{array}$ & $\begin{array}{c}7930000 \\
(7298000-8414000)\end{array}$ & $\begin{array}{c}8712000 \\
(8012000-9244000)\end{array}$ & $\begin{array}{c}9305000 \\
(8550000-9875000)\end{array}$ \\
\hline $\begin{array}{l}\text { Crude all-ages NAFLD } \\
\text { prevalence rate, } \%\end{array}$ & $20.8(19.1-22.1)$ & $21.1(19.4-22.4)$ & $22.2(20.5-23.6)$ & $22.9(21.1-24.3)$ \\
\hline $\begin{array}{l}\text { Adjusted all-ages NAFLD } \\
\text { prevalence rate, } \% \dagger\end{array}$ & $20.8(19.1-22.1)$ & $21.0(19.4-22.3)$ & $22.0(20.2-23.4)$ & $22.3(20.5-23.7)$ \\
\hline Stage F0 & $\begin{array}{c}6436000 \\
(5784000-6956000)\end{array}$ & $\begin{array}{c}6553000 \\
(5884000-7086000)\end{array}$ & $\begin{array}{c}7060000 \\
(6300000-7655000)\end{array}$ & $\begin{array}{c}7400000 \\
(6577000-8048000)\end{array}$ \\
\hline Stage F1 & $630000(425000-872000)$ & 649000 (437 000-899 000) & 736000 (496 000-1 022000$)$ & 807000 (542 000-1 123000$)$ \\
\hline Stage F2 & $360000(235000-508000)$ & 376000 (245 000-530 000) & $451000(298000-636000)$ & 518000 (341 000-730 000) \\
\hline Stage F3 & $216000(131000-316000)$ & 228000 (139 000-334 000) & $293000(179000-424000)$ & $357000(219000-511000)$ \\
\hline Compensated cirrhosis & 101000 (60 400-167 000) & 108000 (65 200-179 000) & 150000 (91 300-242 000) & 195000 (120 000-309 000) \\
\hline $\begin{array}{l}\text { Decompensated cirrhosis, } \\
\text { hepatocellular carcinoma } \\
\text { and liver transplantation }\end{array}$ & $14000(8600-21700)$ & $15100(9300-23500)$ & $21300(13300-33700$ & 28200 (17 700-44 700) \\
\hline $\mathrm{NASH}$ & $\begin{array}{c}1953000 \\
(1582000-2320000)\end{array}$ & $\begin{array}{c}2020000 \\
(1635000-2401000)\end{array}$ & $\begin{array}{c}2345000 \\
(1888000-2792000)\end{array}$ & $\begin{array}{c}2630000 \\
(2107000-3136000)\end{array}$ \\
\hline $\begin{array}{l}\text { Crude all-ages NASH } \\
\text { prevalence rate, } \%\end{array}$ & $5.2(4.2-6.2)$ & $5.4(4.3-6.4)$ & $6.0(4.8-7.1)$ & $6.5(5.2-7.7)$ \\
\hline $\begin{array}{l}\text { Adjusted all-ages NASH } \\
\text { prevalence rate, } \% \dagger\end{array}$ & $5.2(4.2-6.2)$ & $5.3(4.3-6.3)$ & $5.8(4.7-6.9)$ & $6.1(4.9-7.2)$ \\
\hline \multicolumn{5}{|c|}{ Model-estimated incident cases } \\
\hline Decompensated cirrhosis & $3400(1900-5800)$ & $3700(2100-6200)$ & $5100(2900-8500)$ & $6700(3800-10900)$ \\
\hline Hepatocellular carcinoma & $660(440-990)$ & $710(470-1100)$ & $940(620-1400)$ & $1200(770-1800)$ \\
\hline Liver death & $2700(1500-4500)$ & $2900(1700-4800)$ & $4200(2400-6800)$ & $5600(3200-9000)$ \\
\hline
\end{tabular}

Even greater relative increases are projected in later disease stages: the estimated number of stage $\mathrm{F} 2$ cases would increase by $45 \%$, the estimated number of stage $\mathrm{F} 3$ cases would increase by $65 \%$, and the estimated number of compensated cirrhosis cases would increase by $95 \%$.

The number of estimated prevalent cases classified as hepatocellular carcinoma, decompensated cirrhosis or liver transplantation related to NAFLD would increase the most, by $100 \%$, to 28200 (17 700-44 700).

\section{Nonalcoholic steatohepatitis}

The number of prevalent NASH cases would increase by $35 \%$ between 2019 and 2030, to 2630000 (2 107 000-3 136 000) (Figure 1). Among the total NAFLD population, 25.2\% of cases were projected to have NASH in 2019, increasing to $28.3 \%$ in 2030 . The crude all-ages prevalence of NASH would increase from $5.2 \%(4.2 \%-6.2 \%)$ to $6.5 \%(5.2 \%-$ $7.7 \%)$, and the age-adjusted prevalence would increase from $5.2 \%(4.2 \%-6.2 \%)$ to $6.1 \%(4.9 \%-7.2 \%)$ (Table 1$)$.

Of NASH cases in 2019,349000 would be estimated to be stage F3-F4 fibrosis, decompensated cirrhosis, hepato- cellular carcinoma or liver transplantation, encompassing $17 \%$ of estimated NASH cases and $0.9 \%$ of the Canadian population (all ages). By 2030, this number would be expected to increase by $65 \%$, to 578000 cases, and would account for $22 \%$ of predicted NASH cases and $1.0 \%$ of the total population.

\section{Decompensated cirrhosis and hepatocellular carcinoma related to nonalcoholic fatty liver disease} Estimated incident cases of decompensated cirrhosis would almost double between 2019 and 2030, from 3400 (1900$5800)$ in 2019 to $6700(3800-10900)$ in 2030 (Figure 2). The modelled cumulative incidence during the period would number 59800 cases. The estimated number of incident hepatocellular carcinoma cases would increase by $80 \%$, from 660 (440-990) to $1200(770-1800)$, and the modelled cumulative incidence would number 11000 cases. Estimated incident hepatocellular carcinoma was compared with reported estimates for 1992-2010, and model results were within reported ranges (Appendix 1, Supplemental Figure S4). 


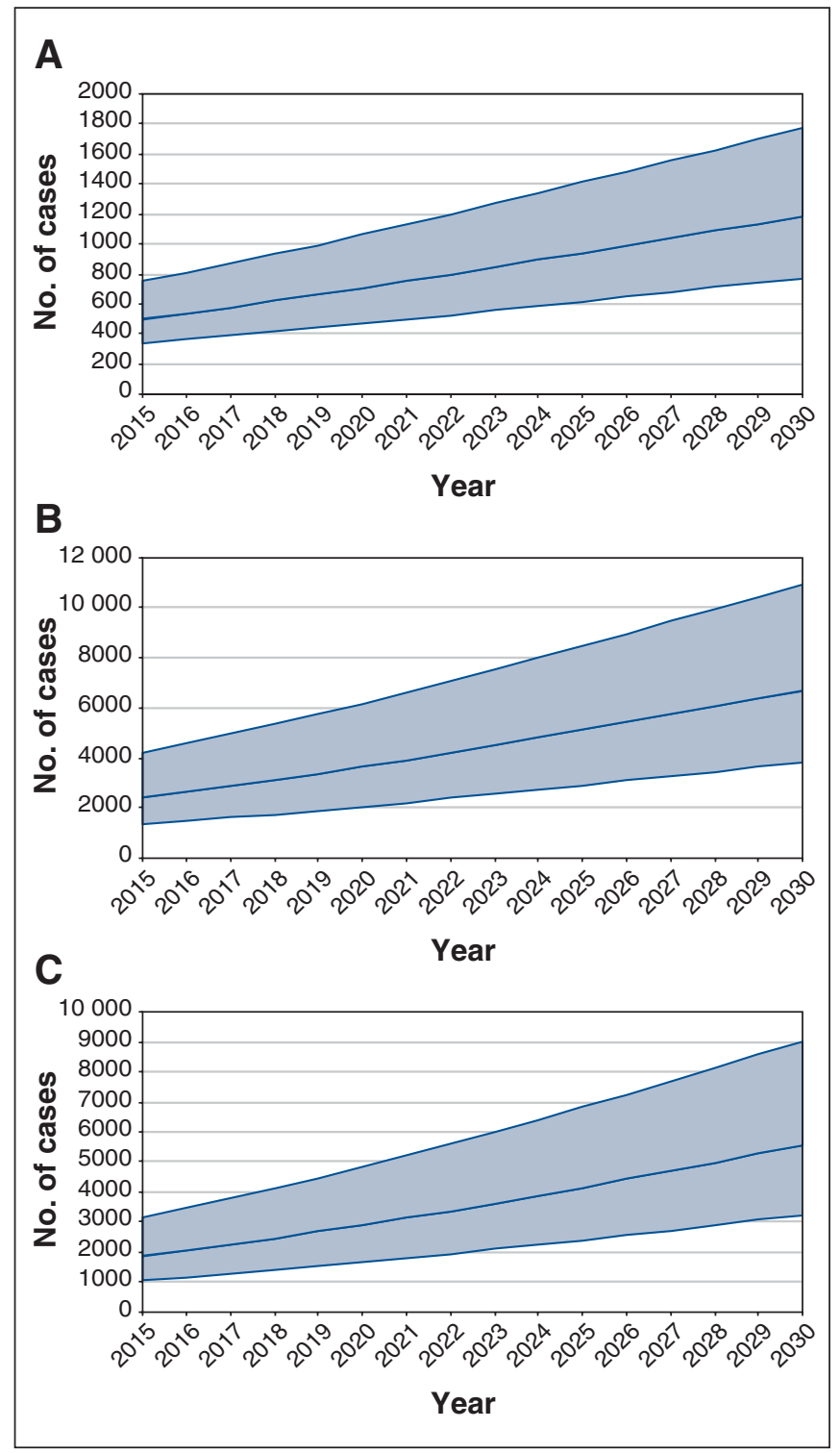

Figure 2: Model-estimated incident cases of hepatocellular carcinoma (A), decompensated cirrhosis (B) and incident liver-related death $(C)$ related to nonalcoholic fatty liver disease for Canada, 2015-2030. Shaded areas represent 95\% uncertainty interval.

\section{Mortality}

Over the study period, the estimated number of incident liver-related deaths in the total NAFLD population would double, from 2700 (1500-4500) to 5600 (3200-9000) (Figure 2), and the cumulative number of liver-related deaths would number 48700 . The total estimated number of deaths in the NAFLD population would increase by $75 \%$, from 66100 to 115000 . Estimated liver-related mortality would account for $4.0 \%$ of deaths in 2019 and $4.8 \%$ of deaths in 2030. Among the NASH population, the number of annual deaths was estimated to be 24400 in 2019, doubling to 49100 in 2030. Estimated liver-related death in the NASH population was estimated to account for $10.9 \%$ of total deaths in 2019 and $11.3 \%$ in 2030 .

\section{Uncertainty and sensitivity analysis}

The leading drivers of uncertainty for the number of estimated NASH cases in 2030 included ranges around the rate of transition from stage $\mathrm{F} 0$ to stage $\mathrm{F} 1$, the starting prevalence among adults aged 20 years or more in 2018, and the standard mortality ratios applied to background mortality rates (Appendix 1, Supplemental Figure S5).

\section{Interpretation}

The current analysis shows that NAFLD may represent a growing burden on the Canadian health care system over the next decade. The population of Canada is aging ${ }^{17}$ and will be prone to increased disease progression to advanced fibrosis in the coming years.

Model estimates from this analysis are similar to projections for other countries where the same modelling was applied. ${ }^{13,15}$ Differences between countries in changes in disease burden are a result of different length of model study period, changes in the background population (population change and aging), and the timing and magnitude of the obesity epidemic in each country.

Other investigators have applied a modelling framework to consider the economic impact of NAFLD, including qualityadjusted life-years. ${ }^{8}{ }^{814} \mathrm{~A}$ large portion of estimated costs were among patients with simple steatosis, who were the largest estimated group in the current analysis. However, it is unknown what proportion of patients with simple steatosis are diagnosed, under care and incurring costs, given the burden of occult liver disease, including compensated cirrhosis. ${ }^{37}$

Estimation of quality-adjusted life-years is an important measure for estimating disease burden, but challenges exist for understanding NAFLD in this context. Given the common comorbidities among patients with NAFLD,${ }^{38}$ decreased quality of life may be negligible, as utility measures are not additive. ${ }^{39}$ Future research should focus on the burden of NAFLD in terms of quality-adjusted life-years, with attention to advanced cases and adjustment for comorbidities.

Challenges exist for modelling based on health records. Chronic liver disease, even cirrhosis, may go unrecognized in primary care,${ }^{37}$ but algorithms exist for identifying advanced cases. ${ }^{40}$ Recorded hospital admissions for NAFLD increased sharply during 2007-2014 in the United States; ${ }^{41}$ however, varied diagnostic modalities with different sensitivity levels, along with increased awareness, likely contributed to an unknown degree. Mortality data are subject to unusable or insufficient coding of cause of death, ${ }^{42}$ and cause of death in NAFLD cases may be coded as complications of cirrhosis or other complications of metabolic syndrome. ${ }^{43}$ Rates of mortality related to cardiovascular disease and nonliver cancers ${ }^{44-48}$ are elevated in NAFLD cases, and the relation between these comorbid conditions and elevated mortality risk has not been fully quantified.

The time frame of our analysis aligns with 2030 targets to reduce mortality from noncommunicable disease by one-third. ${ }^{49}$ Interventions for obesity will reduce NAFLD disease burden. They should follow a multipronged approach, including 
"1) health services and clinical interventions that target individuals, 2) community-level intervention to influence behaviours, and 3) public policies that target broad social or environmental determinants. ${ }^{50}$ Lifestyle modification interventions that result in weight loss of more than $10 \%$ can result in resolution of NASH and regression of fibrosis. ${ }^{51,52}$

There are opportunities for intervention among Canadians aged 6-17 years, as one-third were classified as overweight or obese based on the Canada Health Measures Survey, and this rate remained stable between 2004 and $2014 .{ }^{53}$ Without intervention, these people will contribute to an ever-growing disease burden in future decades and may experience progression to advanced disease at an earlier age. ${ }^{54}$

\section{Limitations}

Challenges to understanding NAFLD disease burden include the lack of consistent definitions for measuring prevalence in general populations ${ }^{55}$ and the potential failure to identify a substantial portion of the NAFLD population owing to the lack of simple and widely available diagnostic techniques with high positive predictive value. ${ }^{56}$ Estimates of disease burden are usually based on historical data, which may not represent the current situation in an epidemic of increasing obesity, especially among younger people. ${ }^{57} \mathrm{~A}$ lack of consistent diagnostic measures means that reported NAFLD prevalence rates vary between studies, ${ }^{11}$ with NAFLD diagnosis often considered an incidental diagnosis when steatosis is found on ultrasonography. ${ }^{58}$ Longitudinal studies of disease using noninvasive measures in general populations ${ }^{59-63}$ should inform modelling, as in such studies more data are reported for longer periods and noninvasive measures are refined.

We calculated the number of new NAFLD cases over time using changes in obesity, classified on the basis of body mass index. Although waist circumference has been shown to be a better measure of visceral fat, ${ }^{64}$ which is central to the development of NAFLD, measures at the national level are available for limited time points, ${ }^{65}$ which makes comparisons problematic. We ultimately selected body mass index because of the availability of nationally representative longitudinal data over several decades. However, it is important to note that trends using body mass index may not capture changes in the burden of disease in people with normal body mass index who are metabolically unhealthy, among whom the prevalence of NAFLD has been estimated to be $7 \%$ in the $\mathrm{US}^{66}$ and $19 \%$ in Asia. ${ }^{67}$

We compared liver cancer incidence to model outputs. A limitation of this comparison is that overall liver cancer incidence may be underreported, ${ }^{68,69}$ and levels of underreporting are continuously changing, so that application of historical estimates may result in overestimation when applied to the latest data. There is further uncertainty and changes over time with regard to the cause of liver cancer, with viral hepatitis expected to contribute relatively fewer cases in the future. $^{70}$

There are several uncertainties when considering future liver transplantation, including the overall demand for and availability of donor organs, and changes in disease burden for competing indications, such as viral hepatitis. We assumed that the total annual number of transplantation procedures would remain constant in the future, but there is evidence that the proportion of NAFLD-related transplantation procedures is growing in Western countries. ${ }^{71}$ Nonalcoholic steatohepatitis is also a growing cause of transplantation among patients with hepatocellular carcinoma. ${ }^{72}$ Furthermore, the fact that there are overlapping indications for transplantation means that some transplantation procedures for other primary indications may also be related to NAFLD but not identified as such.

Our model excluded data on comorbidities that are confounded with NAFLD, including diabetes and dyslipidemia, ${ }^{38}$ to adjust model inputs. However, we applied excess background mortality to adjust for competing mortality risks among NAFLD cases. Finally, we did not adjust base prevalence and disease progression for ethnicity, although this factor may be important for understanding disease burden. ${ }^{38}$

\section{Conclusion}

Nonalcoholic fatty liver disease may represent a growing burden on the Canadian health care system over the next decade. Increasing rates of obesity, in combination with an aging population, translate into increasing NAFLD-related cases of cirrhosis, hepatocellular carcinoma and related mortality. Prevention efforts should be aimed at reducing the incidence of NAFLD and slowing fibrosis progression among those already affected.

\section{References}

1. Chalasani N, Younossi Z, Lavine JE, et al.; American Gastroenterological Association; American Association for the Study of Liver Diseases; American College of Gastroenterology. The diagnosis and management of non-alcoholic fatty liver disease: practice guideline by the American Gastroenterological Association, American Association for the Study of Liver Diseases, and American College of Gastroenterology. Gastroenterology 2012;142:1592-609.

2. Younossi ZM, Otgonsuren M, Henry L, et al. Association of nonalcoholic fatty liver disease (NAFLD) with hepatocellular carcinoma (HCC) in the United States from 2004 to 2009. Hepatology 2015;62:1723-30.

3. Sanyal A, Poklepovic A, Moyneur E, et al. Population-based risk factors and resource utilization for HCC: US perspective. Curr Med Res Opin 2010;26: 2183-91.

4. Loomba R, Sanyal AJ. The global NAFLD epidemic. Nat Rev Gastroenterol Hepatol 2013;10:686-90.

5. Vogel L. Overweight or overfat? Many Canadians are both. CMAf 2017;189: E1202-3.

6. Fatty liver disease. Toronto: Canadian Liver Foundation; 2017. Available: www.liver.ca/patients-caregivers/liver-diseases/fatty-liver-disease/ (accessed 2020 Mar. 26).

7. Angulo P, Hui JM, Marchesini G, et al. The NAFLD fibrosis score: a noninvasive system that identifies liver fibrosis in patients with NAFLD. Hepatology 2007;45:846-54.

8. GBD 2015 Obesity Collaborators; Afshin A, Forouzanfar MH, Reitsma MB, et al. Health effects of overweight and obesity in 195 countries over 25 years. $N$ Engl 7 Med 2017;377:13-27.

9. Younossi ZM, Henry L. Economic and quality-of-life implications of nonalcoholic fatty liver disease. Pharmacoeconomics 2015;33:1245-53.

10. Younossi ZM, Zheng L, Stepanova M, et al. Trends in outpatient resource utilizations and outcomes for Medicare beneficiaries with nonalcoholic fatty liver disease. 7 Clin Gastroenterol 2015;49:222-7.

11. Younossi ZM, Koenig AB, Abdelatif D, et al. Global epidemiology of nonalcoholic fatty liver disease-Meta-analytic assessment of prevalence, incidence, and outcomes. Hepatology 2016;64:73-84.

12. Wells MM, Li Z, Addeman B, et al. Computed tomography measurement of hepatic steatosis: prevalence of hepatic steatosis in a Canadian population. Can 7 Gastroenterol Hepatol 2016;2016:4930987.

13. Estes C, Anstee QM, Arias-Loste MT, et al. Modeling NAFLD disease burden in China, France, Germany, Italy, Japan, Spain, United Kingdom, and United States for the period 2016-2030. F Hepatol 2018;69:896-904. 
14. Younossi ZM, Blissett D, Blissett R, et al. The economic and clinical burden of nonalcoholic fatty liver disease in the United States and Europe. Hepatology 2016;64:1577-86.

15. Estes C, Razavi H, Loomba R, et al. Modeling the epidemic of nonalcoholic fatty liver disease demonstrates an exponential increase in burden of disease. Hepatology 2018;67:123-33.

16. Kleiner DE, Brunt EM, Van Natta M, et al.; Nonalcoholic Steatohepatitis Clinical Research Network. Design and validation of a histological scoring system for nonalcoholic fatty liver disease. Hepatology 2005;41:1313-21.

17. World population prospects: the 2017 revision. New York: Department of Economic Social Affairs, United Nations; 2017.

18. Hagström H, Nasr P, Ekstedt M, et al. Fibrosis stage but not NASH predicts mortality and time to development of severe liver disease in biopsy-proven NAFLD. 7 Hepatol 2017;67:1265-73.

19. Dulai PS, Singh S, Patel J, et al. Increased risk of mortality by fibrosis stage in nonalcoholic fatty liver disease: systematic review and meta-analysis. Hepatology 2017;65:1557-65.

20. Sanyal AJ, Banas C, Sargeant C, et al. Similarities and differences in outcomes of cirrhosis due to nonalcoholic steatohepatitis and hepatitis C. Hepatology 2006;43:682-9.

21. Rahman RN, Ibdah JA. Nonalcoholic fatty liver disease without cirrhosis is an emergent and independent risk factor of hepatocellular carcinoma: a population based study. Hepatology 2012;56:241A.

22. Ries LAG, Young JL, Keel GE, et al., editors. SEER survival monograph: cancer survival among adults - U.S. SEER program, 1988-2001, patient and tumor characteristics. SEER Program, NIH publ no 07-6215. Bethesda (MD): National Cancer Institute; 2007.

23. Nakanishi R, Li D, Blaha MJ, et al. All-cause mortality by age and gender based on coronary artery calcium scores. Eur Heart 7 Cardiovasc Imaging 2016; 17:1305-14.

24. NCD Risk Factor Collaboration (NCD-RisC). Worldwide trends in bodymass index, underweight, overweight, and obesity from 1975 to 2016: a pooled analysis of 2416 population-based measurement studies in 128.9 million children, adolescents, and adults. Lancet 2017;390:2627-42.

25. NCD Risk Factor Collaboration (NCD-RisC). Trends in adult body-mass index in 200 countries from 1975 to 2014: a pooled analysis of 1698 populationbased measurement studies with 19.2 million participants. Lancet 2016; 387:1377-96.

26. Country profile: Canada — body mass index. NCD Risk Factor Collaboration (NCD-RisC). Available: www.ncdrisc.org/downloads/country-pdf/country -profile-Canada.pdf (accessed 2018 Mar. 18).

27. Deurenberg P, Yap M, van Staveren WA. Body mass index and percent body fat: a meta analysis among different ethnic groups. Int 7 Obes Relat Metab Disord 1998;22:1164-71.

28. Chiu M, Austin PC, Manuel DG, et al. Deriving ethnic-specific BMI cutoff points for assessing diabetes risk. Diabetes Care 2011;34:1741-8.

29. Census profile, 2016 census. Ottawa: Statistics Canada; modified 2019 Aug. 9. Available: www12.statcan.gc.ca/census-recensement/2016/dp-pd/prof/details/ page.cfm?Lang=E\&Geo1=PR\&Code1=01\&Geo2=PR\&Code2=01\&Data=Count $\&$ Search Text $=$ canada $\&$ Search Type $=$ Begins $\&$ SearchPR $=01 \& B 1=A l l \&$ TABID $=1$ (accessed 2019 Jan. 15).

30. Lazo M, Hernaez R, Eberhardt MS, et al. Prevalence of nonalcoholic fatty liver disease in the United States: the Third National Health and Nutrition Examination Survey, 1988-1994. Am 7 Epidemiol 2013;178:38-45.

31. Choi YJ, Lee DH, Han KD, et al. Is nonalcoholic fatty liver disease associated with the development of prostate cancer? A nationwide study with 10,516,985 Korean men. PLoS One 2018;13:e0201308.

32. Caballería L, Pera G, Auladell MA, et al. Prevalence and factors associated with the presence of nonalcoholic fatty liver disease in an adult population in Spain. Eur 7 Gastroenterol Hepatol 2010;22:24-32.

33. Fan JG, Farrell GC. Epidemiology of non-alcoholic fatty liver disease in China. F Hepatol 2009;50:204-10.

34. Shaheen AA, Riazi K, Medellin A, et al. Implementation of a primary care shear-wave elastography-based pathway to identify non-alcoholic fatty liver disease patients with advanced fibrosis in a large North American urban population. F Hepatol 2019;70(Suppl):E783.

35. Organ donation and transplantation in Canada: system progress report 20062015. Donation and Transplantation, Canadian Blood Services; 2016. Available: https://blood.ca/sites/default/files/ODT_Report.pdf (accessed 2019 Oct. 16).

36. Treatment of end-stage organ failure in Canada, Canadian Organ Replacement Register, 2006 to 2015: data tables, liver transplants. Ottawa: Canadian Institute for Health Information. Available: www.cihi.ca/sites/default/files/ document/liver_transplant_section_v0.1_en_2017.xlsx (accessed 2019 Oct. 16).

37. Martini A, Ceranto E, Gatta A, et al. Occult liver disease burden: analysis from a large general practitioners' database. United European Gastroenterol 7 2017;5: 982-6.

38. The diagnosis and management of nonalcoholic fatty liver disease: practice guidance from the American Association for the Study of Liver Diseases. Clin Liver Dis (Hoboken) 2018;11:81.

39. Fu AZ, Kattan MW. Utilities should not be multiplied: evidence from the preference-based scores in the United States. Med Care 2008;46:984-90.
40. Lapointe-Shaw L, Georgie F, Carlone D, et al. Identifying cirrhosis, decompensated cirrhosis and hepatocellular carcinoma in health administrative data: a validation study. PLoS One 2018;13:e0201120.

41. Adejumo AC, Samuel GO, Adegbala OM, et al. Prevalence, trends, outcomes, and disparities in hospitalizations for nonalcoholic fatty liver disease in the United States. Ann Gastroenterol 2019;32:504-13.

42. Mikkelsen L, Iburg KM, Adair T, et al. Assessing the quality of cause of death data in six high-income countries: Australia, Canada, Denmark, Germany, Japan and Switzerland. Int 7 Public Health 2020;65:17-28.

43. Tapper EB, Parikh ND. Mortality due to cirrhosis and liver cancer in the United States, 1999-2016: observational study. BM7 2018;362:k2817.

44. Stepanova M, Rafiq N, Makhlouf H, et al. Predictors of all-cause mortality and liver-related mortality in patients with non-alcoholic fatty liver disease (NAFLD). Dig Dis Sci 2013;58:3017-23.

45. Byrne CD, Targher G. NAFLD: a multisystem disease. 7 Hepatol 2015;62 (Suppl):S47-64.

46. Targher G, Byrne CD, Lonardo A, et al. Nonalcoholic fatty liver disease and risk of incident cardiovascular disease: a meta-analysis of observational studies. 7 Hepatol 2016;65:589-600.

47. Nseir W, Abu-Rahmeh Z, Tsipis A, et al. Relationship between non-alcoholic fatty liver disease and breast cancer. Isr Med Assoc 7 2017;19:242-5.

48. Harding JL, Shaw JE, Anstey KJ, et al. Comparison of anthropometric measures as predictors of cancer incidence: a pooled collaborative analysis of 11 Australian cohorts. Int 7 Cancer 2015;137:1699-708.

49. Global action plan for the prevention and control of noncommunicable diseases: 2013 2020. Geneva: World Health Organization; 2013.

50. Obesity in Canada - opportunities for intervention. Ottawa: Public Health Agency of Canada; 2011 June 23. Available: www.canada.ca/en/public-health/services/health -promotion/healthy-living/obesity-canada/opportunities-intervention.html (accessed 2020 Mar. 20).

51. Romero-Gómez M, Zelber-Sagi S, Trenell M. Treatment of NAFLD with diet, physical activity and exercise. 7 Hepatol 2017;67:829-46.

52. Vilar-Gomez E, Martinez-Perez Y, Calzadilla-Bertot L, et al. Weight loss through lifestyle modification significantly reduces features of nonalcoholic steatohepatitis. Gastroenterology 2015;149:367-78.e5; quiz e14-5.

53. Rao DP, Kropac E, Do MT, et al. Childhood overweight and obesity trends in Canada. Health Promot Chronic Dis Prev Can 2016;36:194-8.

54. Ratziu V, Marchesini G. When the journey from obesity to cirrhosis takes an early start. 7 Hepatol 2016;65:249-51.

55. European Association for Study of Liver; Asociacion Latinoamericana para el Estudio del Higado. EASL-ALEH Clinical Practice Guidelines: non-invasive tests for evaluation of liver disease severity and prognosis. 7 Hepatol 2015;63: 237-64.

56. European Association for the Study of the Liver (EASL); European Association for the Study of Diabetes (EASD); European Association for the Study of Obesity (EASO). EASL-EASD-EASO Clinical Practice Guidelines for the management of non-alcoholic fatty liver disease. Diabetologia 2016;59:1121-40.

57. Alberti G, Zimmet P, Shaw J, et al.; Consensus Workshop Group. Type 2 diabetes in the young: the evolving epidemic: the International Diabetes Federation consensus workshop. Diabetes Care 2004;27:1798-811.

58. Wilkins T, Tadkod A, Hepburn I, et al. Nonalcoholic fatty liver disease: diagnosis and management. Am Fam Physician 2013;88:35-42.

59. Kabbany MN, Conjeevaram Selvakumar PK, Watt K, et al. Prevalence of nonalcoholic steatohepatitis-associated cirrhosis in the United States: an analysis of National Health and Nutrition Examination Survey data. Am 7 Gastroenterol 2017;112:581-7.

60. Kim D, Kim W, Adejumo AC, et al. Race/ethnicity-based temporal changes in prevalence of NAFLD-related advanced fibrosis in the United States, 2005 2016. Hepatol Int 2019;13:205-13.

61. Petta S, Di Marco V, Pipitone RM, et al. Prevalence and severity of nonalcoholic fatty liver disease by transient elastography: genetic and metabolic risk factors in a general population. Liver Int 2018;38:2060-8.

62. Caballería L, Pera G, Arteaga I, et al. High prevalence of liver fibrosis among European adults with unknown liver disease: a population-based study. Clin Gastroenterol Hepatol 2018;16:1138-45.e5.

63. Wong VW, Chu WC, Wong GL, et al. Prevalence of non-alcoholic fatty liver disease and advanced fibrosis in Hong Kong Chinese: a population study using proton-magnetic resonance spectroscopy and transient elastography. Gut 2012; 61:409-15.

64. Balakrishnan M, El-Serag HB, Nouyen T, et al. Obesity and risk of nonalcoholic fatty liver disease: a comparison of bioelectrical impedance analysis and conventionally-derived anthropometric measures. Clin Gastroenterol Hepatol 2017;15:1965-7

65. Shields M, Tremblay MS, Connor Gorber S, et al. Measures of abdominal obesity within body mass index categories, 1981 and 2007-2009. Health Rep 2012;23:33-8.

66. Younossi ZM, Stepanova M, Negro F, et al. Nonalcoholic fatty liver disease in lean individuals in the United States. Medicine (Baltimore) 2012;91:319-27.

67. Fan JG, Kim SU, Wong VW. New trends on obesity and NAFLD in Asia. 7 Hepatol 2017;67:862-73.

68. Törner A, Stokkeland K, Svensson A, et al. The underreporting of hepatocellular carcinoma to the cancer register and a log-linear model to estimate a more correct incidence. Hepatology 2017;65:885-92. 
69. Hong TP, Gow P, Fink M, et al. Novel population-based study finding higher than reported hepatocellular carcinoma incidence suggests an updated approach is needed. Hepatology 2016;63:1205-12.

70. Report on hepatitis $B$ and $C$ in Canada: 2017. Centre for Communicable Diseases and Infection Control, Infectious Disease Prevention and Control Branch, Public Health Agency of Canada; 2019. Cat no HP37-22E-PDF. Available: https://www.canada.ca/en/services/health/publications/diseases -conditions/report-hepatitis-b-c-canada-2017.html\#a4.1 (accessed 2020 May 26).

71. Wong RJ, Cheung R, Ahmed A. Nonalcoholic steatohepatitis is the most rapidly growing indication for liver transplantation in patients with hepatocellular carcinoma in the U.S. Hepatology 2014;59:2188-95.

72. Younossi Z, Stepanova M, Ong JP, et al.; Global Nonalcoholic Steatohepatitis Council. Nonalcoholic steatohepatitis is the fastest growing cause of hepatocellular carcinoma in liver transplant candidates. Clin Gastroenterol Hepatol 2019; 17:748-55.e3.

Affiliations: Division of Gastroenterology and Hepatology (Swain, Shaheen), Cumming School of Medicine, University of Calgary, Calgary, Alta.; Division of Gastroenterology (Ramji), University of British Columbia, Vancouver, BC; Toronto Centre for Liver Disease (Patel), University Hospital Network, Toronto, Ont.; Division of Gastroenterology and Hepatology (Sebastiani), McGill University Health Centre, Montréal, Que.; LAIR Centre (Tam), Vancouver, BC; Division of Gastroenterology (Marotta), Department of Medicine, Schulich School of Medicine and Dentistry, University of Western Ontario, London, Ont.; Toronto Liver
Centre (Elkhashab), Toronto, Ont.; LMC Diabetes and Endocrinology Brampton (Bajaj), Brampton, Ont.; Leadership Sinai Centre for Diabetes (Bajaj), Mount Sinai Hospital, Toronto, Ont.; Center for Disease Analysis Foundation (Estes, Razavi), Lafayette, Colo.

Contributors: Homie Razavi and Chris Estes conceived and designed the study. All of the authors contributed data, analyzed and interpreted the data, drafted the manuscript and revised it critically for important intellectual content, approved the final version to be published and agreed to be accountable for all aspects of the work.

Funding: Funding for this project was provided by Gilead Sciences. Giada Sebastiani is supported by Junior 1 and 2 Salary Awards (27127 and 267806) from the Fonds de recherche du Québec - Santé and research salary from the Department of Medicine of McGill University.

Data sharing: Model results presented in the current analysis are available by contacting info@cdafound.org.

Disclaimer: The funders had no role in the study design, data collection, analysis or interpretation of data, or preparation of the manuscript.

Supplemental information: For reviewer comments and the original submission of this manuscript, please see www.cmajopen.ca/content/8/2/ E429/suppl/DC1. 